\title{
Level of Certain Oxidants and Antioxidants in Patients with Uterine Fibroids
}

\author{
Kadir CETINKAYA ${ }^{1,2}$, Pelin ARIBAL AYRAL ${ }^{2}$, Semra CETINKAYA ${ }^{2,3}$, Nuray YAZIHAN ${ }^{2}$, Seyma FADILOGLU ${ }^{4}$, \\ Melahat ATASEVER ${ }^{5}$, Ozcan EREL ${ }^{6}$
}

Ankara, Turkey

ABSTRACT

OBJECTIVE: The mechanism of the leiomyoma formation process is still unknown. However, the menstrual cycle is associated with hypoxia, and ongoing hypoxia is associated with cellular events leading to the conversion of myometrial cells into uterine fibroids. After the hypoxic environment, the balance of cellular cytokines and growth factors changes. It was planned to evaluate the balance of oxidative agents and the role of oxidative stress in the formation of uterine fibroids.

STUDY DESIGN: The study was conducted in patients of gynecology outpatient clinic of Ankara Oncology Training and Research Hospital. Those volunteers with fibroids formed the study group, and the healthy ones were identified as the control group. A total of 64 subjects, 32 in both groups were included. The levels of catalase, ceruloplasmin, myeloperoxidase, native thiol, disulfide, and total thiol levels were investigated in groups.

RESULTS: Antioxidant parameters such as native thiol, disulfide, and total thiol levels were significantly decreased in the uterine fibroid group. Catalase, ceruloplasmin, and myeloperoxidase levels were not statistically different between groups.

CONCLUSION: Decrease in these antioxidant parameters showed that the hypoxia and the balance of oxidant and antioxidant substances changes may probably have aociated with the pathophysiology of uterine fibroids.

Keywords: Antioxidants, Oxidants, Oxidative stre, Pathophysiology, Uterine fibroid

Gynecol Obstet Reprod Med 2019;25(3):158-162

1 Department of Obstetrics and Gynecology, Ankara Oncology Training and Research Hospital, Ankara, Turkey

2 Department of Internal Medicine, Division of Pathophysiology, Ankara University, Ankara Turkey

3 Department of Pediatrics, Health Sciences University, Ankara Turkey

4 Department of Obstetrics and Gynecology, Ankara Numune Training and Research Hospital, Ankara, Turkey

5 Department of Obstetrics and Gynecology, Giresun University, Giresun, Turkey

6 Department of Biochemistry, Ankara Yildirim Beyazid University, Ankara, Turkey

Address of Correspondence: Kadir Cetinkaya

Ankara City Hospital, Department of

Obstetrics and Gynecology,

06800 Ankara, Turkey

kacetinkaya@gmail.com

Submitted for Publication:

17.04.2019

Revised for: Publication: 22.05.2019

Accepted for Publication: $\quad$ 19.07.2019

ORCID IDs of the authors: KC: 0000-0003-4490-8267,

PAA:0000-0002-5047-7881, SC: 0000-0003-3974-2872,

NY: 0000-0003-1237-8468, SF: 0000-0001-8228-3515

MA:0000-0001-8232-4719, OE: 0000-0002-2996-3236

\begin{tabular}{|c|c|}
\hline Quick Response Code: & Access this article online \\
\cline { 2 - 2 } & Website: www.gorm.com.tr \\
e- mail: infogorm.com.tr
\end{tabular}

How to cite this article: Cetinkaya K. Aribal Ayral P. Cetinkaya S. Yazihan N. Fadiloglu S. Atasever M. Erel O. Level of Certain Oxidants and Antioxidants in Patients with Uterine Fibroids Gynecol Obstet Reprod Med. 2019;25 (3): $158-162$

\section{Introduction}

Uterine fibroids are benign smooth muscle tumors originating from myometrium, containing various amounts of fibrous tissue. The incidence of uterine fibroids observed in the reproductive age of women over 30 years of age is between $30 \%$ and $70 \%$ (1). In many women, uterine fibroids are clinically insignificant and asymptomatic. However, their number, size, and placement in the uterus may cause various symptoms. Although most of them do not produce any signs, up to $20 \%$ may cause excessive and irregular bleeding, anemia, pelvic pain, bowel and bladder dysfunction, and pain symptoms during sexual intercourse (2). In addition, uterine fibroids are also associated with recurrent pregnancy losses or infertility (3).

Uterine fibroids with different clinical presentations and which may cause serious symptoms can cause significant morbidity, the cost for the health system, reduce the quality of life for many patients, and can lead to a need for treatment including surgical intervention.

For these reasons, considering the high prevalence, it constitutes an important part of daily gynecology practice. Considering the causes of hysterectomy, uterine fibroids are the leading indications. According to the clinical findings of the 
patients, non-surgical treatment methods that can be recommended including follow-up, medical treatments or radiological interventions. Therefore, the discovery of new treatment methods or preventive mechanisms that will remove them from surgical treatment will benefit public health in all respects.

Examining the etiologic and pathophysiological risk factors, endogenous and exogenous estrogen exposure is at the forefront and constitutes the basis of many treatment modalities. However, chromosomal anomalies and the events related to the intracellular metabolism are thought to play a role in the formation of uterine fibroids (4). In studies on intracellular pathways, the microenvironment was more hypoxic in fibroids compared to normal myometrium, and antioxidant enzyme systems were observed to be deteriorated (4). All of these are thought to play a role in the pathophysiology of uterine fibroids and are the subject of many scientific studies.

In this study, we compared the oxidative and antioxidative markers of individuals with and without uterine fibroids. It was planned to evaluate the role of oxidative agents and oxidative stress in the formation of uterine fibroids.

\section{Material and Method}

The study was carried out in a gynecology outpatient clinic of Ankara Oncology Training and Research Hospital. The volunteers of the uterine fibroids group consisted, those who agreed to participate in the study diagnosed as having fibroids, had no other disease, between 18 and 50 years of age and had no menopause. Reproductive age, healthy volunteers were the control group. While fasting venous blood samples were taken during the menstrual period from those patients for their other examinations, $5 \mathrm{~mL}$ of blood samples were taken in a separate biochemistry tube containing ethylenediaminetetraacetic acid (EDTA) from each group.

Serum was separated after centrifugation at $1600 \mathrm{G}$ for 10 minutes. Materials were prepared in the biochemistry laboratory and stored at -80 degrees until the work was finished. A total of 64 subjects, 32 in the study group and 32 in the control group, were included in the study.

The age, menstrual status, medications, family history, and other diseases were questioned.

Exclusion criteria were diabetes mellitus, hyperthyroidism, hypothyroidism, cardiovascular disease, cerebrovascular disease, acute-chronic kidney disease, liver disease, nephrotic-level proteinuria, acute-chronic infection, collagen tissue disease, disorders that could affect metabolic parameters, presence of systemic diseases such as malignancy, oral contraceptive, antioxidant drug, vitamin supplement, lipidlowering medication use, smoking or alcohol use, and pregnancy were considered. Furthermore, any previous medical or surgical treatment related to uterine fibroids was defined as an exclusion criteria. All patients were evaluated via transvaginal ultrasonography in the study period by an experienced gynecologist (KC).

Catalase, myeloperoxidase, ceruloplasmin, native thiol, disulfide, and total thiols were studied in all subjects. Thiol and disulfide homeostasis tests were measured by the automatic spectrophotometric method (5).

Measured values were examined and reported as mean \pm standard deviation, median (min-max) and number. The normality of the distribution of groups was determined with the Kolmogorov-Smirnov test. Parametric data were analyzed by independent two-sample t-test and non-parametric data were compared using the Mann Whitney-U test. A $p$ value $<0.05$ was considered statistically significant.

The ethical approval of this prospective case-control study was obtained from the ethics committee of Ankara Oncology Training and Research Hospital with the approval number of $2016-10 / 220$ on $19^{\text {th }}$ October 2016 . This study was also conducted according to the Declaration of Helsinki and informed consent was obtained from all volunteers.

\section{Results}

The median ages of the subjects were 39 (22-49) and 43 (27-48) for the control group and the study group, respectively, and the mean age was 37.03 and 40.5 for the control and study groups, respectively. The mean diameter of the uterine fibroids was $46.2(31-82) \mathrm{mm}$. Mean gravida values were $2.21 \pm 2.14$ and $3.10 \pm 1.81$ in control and uterine fibroid groups, respectively. Parity mean values were $1.79 \pm 1.42$ and $2.24 \pm 1.04$ in the control and uterine fibroid groups, respectively. There was no significant difference between demographic findings.

The results of oxidative and antioxidative parameters of the levels were summarized in table I.

Native thiol levels were lower in the uterine fibroid group and a significant difference was observed between the uterine fibroid group and the control group (Native thiol levels ( $\mu \mathrm{mol} / \mathrm{L}$ ); uterine fibroid group: $281.63 \pm 53.14$;Control Group; $306.50 \pm 42.92, p=0.044)$.

Total thiol levels were similarly lower in the uterine fibroid group and there was a significant difference between the uterine fibroid group and the control group. (Total thiol ( $\mu \mathrm{mol} / \mathrm{L}$ ); uterine fibroid group: $325.12 \pm 52.56$; Control Group: $359.00 \pm$ 41.07, $p=0.006$ ).

The disulfide parameters were lower in the uterine fibroid group and there was a statistically significant difference with the control group. (Disulfide ( $\mu \mathrm{mol} / \mathrm{L})$; Uterine fibroid Group: 21.74 \pm 7.65; Control Group: 26.24 $\pm 8.69, p=0.032$ ).

Although there were differences between the groups in terms of ceruloplasmin, catalase, and myeloperoxidase, no statistical significance was observed in any category $(p>0.05)$. 
Table I: Levels of oxidative and antioxidative markers

\begin{tabular}{lccc}
\hline Parameter & Uterine fibroid group & Control group & $p$ \\
\hline Ceruloplasmin $(\mathrm{U} / \mathrm{L})$ & $100.21 \pm 23.27$ & $97.76 \pm 33.28$ & 0.735 \\
Catalase $(\mathrm{U} / \mathrm{L})$ & $98.59 \pm 121.31$ & $56.25 \pm 55.58$ & 0.080 \\
Myeloperoxidase $(\mathrm{U} / \mathrm{L})$ & $83.73 \pm 47.91$ & $93.22 \pm 35.64$ & 0.373 \\
$\mathrm{SH}(\mu \mathrm{mol} / \mathrm{L})$ & $281.63 \pm 53.14$ & $306.50 \pm 42.92$ & 0.044 \\
$\mathrm{SS}+\mathrm{SH}(\mu \mathrm{mol} / \mathrm{L})$ & $325.12 \pm 52.56$ & $359.00 \pm 41.07$ & 0.006 \\
$\mathrm{SS}(\mu \mathrm{mol} / \mathrm{L})$ & $21.74 \pm 7.65$ & $26.24 \pm 8.69$ & 0.032 \\
\hline
\end{tabular}

SH: Native thiol, SS+SH: Total thiol, SS: Disülfid

\section{Discussion}

In this study, the thiol-associated antioxidant parameters, native thiol, total thiol and disulfide levels were significantly lower in patients with uterine fibroids. However, there was no significant change in ceruloplasmin, catalase, and myeloperoxidase levels.

The mechanism of the formation of leiomyoma is still unknown. Myometrial stem cells have been shown to be differentiated into mature myometrial cells only under hypoxic conditions in vitro conditions and suggest that hypoxia may be the driving force behind growth and transformation in conversion to uterine fibroid cells $(4,6)$.

Oxidative stress is evaluated as responsible for the etiopathogenesis and pathophysiology of many diseases (7-9). Reactive oxygen species (ROS) production occurs immediately after exposure to oxidative stress and hypoxia. In order to protect the tissues against the harmful effects of ROS, enzymatic or nonenzymatic antioxidant mechanisms are introduced into the circuit (8). In this study, it was aimed to investigate whether some oxidant and antioxidant parameters of individuals with uterine fibroids were changed according to the control group.

Cyclic menstrual contractions of the myometrium result in periodic hypoxia/ischemia, which may lead to the differentiation of the uterine fibroid development of the myometrial stem cells. The continuation of uncontrolled proliferation of mutated stem cell-derived cells results in uterine fibroid foci. The effects of gonadal steroids in combination with chronic hypoxia associated with rapidly expanding leiomyoma cell mass stimulate local angiogenic growth factor expression $(6,10)$. They promote continued cell proliferation and extracellular matrix deposition and provide vascular support to the growing myometrial cell mass and cause uterine fibroids $(4,11,12)$.

Abnormalities and angiogenic growth factors in uterine vessels are thought to play a role in the pathogenesis of uterine fibroids $(4,11,12)$. A myomatous uterus accommodates a large number of enlarged arterioles and venules. Based on the traumatic injury of tissue hypoxia, it is thought that fibroid formation may be secondary to trauma such as atherosclerotic plaque formation secondary to arterial muscle hypoxia (11). Based on this hypothesis, it can be thought that the myometrial cells that remain hypoxic during menstruation will progress to the conversion to abnormal myocytes and this situation strengthens the idea based on the hypoxic mechanism (12).

Oxidative stress is the deterioration of molecular and cellular functions due to the loss of balance between the production of free radical or reactive oxygen species and the antioxidant system (13). It results from the imbalance between free radical production and endogenous antioxidant defense mechanisms. This balance is even shown to be affected by the menstrual period which is regulated by the reproductive system hormones, mainly by estrogen which is known to be having anti-oxidant effects $(14,15)$.

Oxidative stress is related to many diseases and the thiolmediated antioxidant system was found to be weakened in obstetric complications such as fetal growth retardation, preeclampsia, premature ovarian failure, hyperemesis gravidarum (16-18). Antioxidant effect and thiol, which is a part of cysteine amino acid, play a significant role in preventing oxidative stress in the cell (19).

Inhibition of oxidative stress in the cell by amino acids and proteins is an important defense function.

Thiol cells have a critical role in preventing the formation of any oxidative stress state (19). Thiol groups are also among antioxidant mechanisms. Thiols can react with free radicals to protect against tissue and cell damage caused by reactive oxygen products and contain sulfhydryl (-SH) group, an antioxidant molecule $(19,20)$.

Dynamic thiol and disulfide levels are involved in antioxidant defense, apoptosis, regulation of enzyme activities, and cellular signal transduction mechanisms (21).

The thiol groups of sulfur-containing amino acids (cysteine, methionine) in proteins are the primary target point of ROS (22-25).

The resulting disulfide bond structures can be reduced back to the thiol groups, and thus the thiol-disulfide balance is maintained (19).

Uterine fibroids are very common gynecological pathologies and have been tried to be associated with oxidative pathways (26). 
Individuals with uterine fibroids were found to have lower serum thiol levels and higher oxidative protein products compared to individuals in the control group (27). Similar results have been shown in different studies and it has been shown that both oxidative and antioxidative capacities are increased (28).

Preservation of antioxidant balance and oxidative stress are important for a variety of benign and malignant conditions such as diabetes mellitus and the familial Mediterranean fever as well as for uterine fibroids (16,29-31). Relationship between oxidative stress and uterine fibroids was demonstrated in studies regarding surgically removed leiomyomas and found clear interaction between mainly by lipid peroxidation and leiomyoma (32). This oxidative imbalance was also found to be shown in fibroid cells compared to normal fibroid cells which also supports the relationship between oxidative stress and development of leiomyoma (33).

Although the pathophysiological effect of the oxidative agents has been clearly demonstrated, the direct effect of antioxidant treatments on the pathogenesis of the pathways has not been shown so far. Similar findings were observed in the literature about uterine fibroids and oxidative processes, and lipid peroxidation and deterioration in antioxidant enzyme levels were shown in plasma and erythrocyte cells in patients with uterine fibroids $(4,26,34)$.

Patients with uterine fibroids were found to have lower serum thiol levels and higher oxidative protein products compared to the control group. However, when these oxidative agents were tried to be associated with symptoms of uterine fibroids, no significant relationship could be observed (27). Similar results have also been found in many studies and it has been observed that oxidants and antioxidants levels have also changed $(28,35)$.

In the present study, a significant reduction in the levels of thiol-associated antioxidant parameters compared to the control group shows that the results obtained from this study support the findings in the literature. When all these data are interpreted together, it can be seen that oxidative stress may be involved in the pathogenesis of uterine fibroids and especially the thiol-associated pathways may have an effect. The limitation of the study is a relatively small number of participants in the groups and the lack of analysis for the relationship between the size of uterine fibroids and serum levels of markers.

Since thiol can be reduced, any treatment that causes or decreases this reduction may prevent pathophysiological processes resulting from abnormal levels of thiol and disulfide. Therefore, it is important to determine the levels of dynamic thiol and disulfide in diseases where oxidative stress plays an important role in pathogenesis $(4,6,35)$.

The control of oxidative stress caused by these reasons is thought to be effective in the prophylaxis of uterine fibroids. Nowadays, it is known that antioxidant agents and related diet products contribute to the process and have a role in treatment in many pathological conditions (36-38). Considering all these mechanisms, the role of antioxidant agents in the treatment of uterine fibroids can be discussed. These findings may shed light on other studies in the future and will have new potential expansions.

Disclosure Statement: Authors' have nothing to declare and no conflict of interest. This study was not funded by any company or third person.

Author Contributions: KC: Concept, Interpretation, Design, Writer. PAA: Supervision, Concept, Design. SC: Supervision, Design, Concept. NY: Supervision, Concept, Design, Statistical analysis, Data interpretation. SF: Supervision, Concept, Design. MA: Supervision, Concept, Design. OE: Analysis, Data analysis, Data collection, Data processing, Data interpretation

\section{References}

1. Okolo S. Incidence, etiology and epidemiology of uterine fibroids. Best Pract Res Clin Obstet Gynaecol. 2008;22 (4):571-88.

2. Marsh EE, Bulun SE. Steroid hormones and leiomyomas. Obstet Gynecol Clin North Am. 2006;33(1):59-67.

3. Eldar-Geva T, Meagher S, Healy DL, MacLachlan V, Breheny S, Wood C. Effect of intramural, subserosal, and submucosal uterine fibroids on the outcome of assisted reproductive technology treatment. Fertil Steril. 1998;70 (4):687-91.

4. Tal R, Segars JH. The role of angiogenic factors in fibroid pathogenesis: potential implications for future therapy. Hum Reprod Update. 2014;20(2):194-216.

5. Erel O, Neselioglu S. A novel and automated assay for thiol/disulphide homeostasis. Clin Biochem. 2014;47 (18):326-32.

6. Borahay MA, Al-Hendy A, Kilic GS, Boehning D. Signaling pathways in leiomyoma: understanding pathobiology and implications for therapy. Mol Med. 2015; $21: 242-56$

7. Eren Y, Dirik E, Neşelioğlu S, Erel Ö. Oxidative stress and decreased thiol level in patients with migraine: cross-sectional study. Acta Neurol Belg. 2015;115(4):643-9.

8. Başkol M, Dolbun Seçkin K, Başkol G. Advanced oxidation protein products, total thiol levels and total oxidant/antioxidant status in patients with nash. Turk J Gastroenterol. 2014;25 Suppl 1:32-7.

9. Karadag-Oncel E, Erel O, Ozsurekci Y, Caglayik DY, Kaya A, Gozel MG, et al. Plasma oxidative stress and total thiol levels in Crimean-Congo hemorrhagic fever. Jpn J Infect Dis. 2014;67(1):22-6.

10. Torres-de la Roche LA, Becker S, Cezar C, Hermann A, Larbig A, Leicher L, et al. Pathobiology of myomatosis uteri: the underlying knowledge to support our clinical 
practice. Arch Gynecol Obstet. 2017;296(4):701-7.

11. Stewart EA, Nowak RA. New concepts in the treatment of uterine leiomyomas. Obstet Gynecol. 1998;92(4 Pt 1): 624-7.

12. Faerstein E, Szklo M, Rosenshein NB. Risk factors for uterine leiomyoma: a practice-based case-control study. II. Atherogenic risk factors and potential sources of uterine irritation. Am J Epidemiol. 2001;153(1):11-9.

13. Ames BN, Shigenaga MK, Hagen TM. Oxidants, antioxidants, and the degenerative diseases of aging. Proc Natl Acad Sci U S A. 1993;90(17):7915-22.

14. Cornelli U, Belcaro G, Cesarone MR, Finco A. Analysis of oxidative stress during the menstrual cycle. Reprod Biol Endocrinol. 2013;11:74.

15. Karowicz-Bilinska A, Plodzidym M, Krol J, Lewinska A, Bartosz G. Changes of markers of oxidative stress during menstrual cycle. Redox Rep. 2008;13(5):237-40.

16. Cakar E, Ayvacı H, Karcaaltincaba D, Aydın G, Cilli A, Bicer C, et al. Thiol-disulfide homoestasis in pregnancies with fetal growth restriction. J Matern Fetal Neonatal Med. 2018;11:1-6.

17. Isik H, Sahbaz A, Timur H, Aynioglu O, Atalay Mert S, Balık AR, et al. The use of thiol/disulfide as a novel marker in premature ovarian failure. Gynecol Obstet Invest. 2017;82(2):113-8.

18. Ergin M, Cendek BD, Neselioglu S, Avsar AF, Erel O. Dynamic thiol-disulfide homeostasis in hyperemesis gravidarum. J Perinatol. 2015;35(10):788-92.

19. Go YM, Jones DP. Thiol/disulfide redox states in signaling and sensing. Crit Rev Biochem Mol Biol. 2013;48 (2):173-81.

20. Gulpamuk B, Tekin K, Sonmez K, Inanc M, Neselioglu S, Erel $\mathrm{O}$, et al. The significance of thiol/disulfide homeostasis and ischemia-modified albumin levels to assess the oxidative stress in patients with different stages of diabetes mellitus. Scand J Clin Lab Invest. 2018;78(1-2):136-42.

21. Sies H, Stahl W, Sundquist AR. Antioxidant functions of vitamins. Vitamins $\mathrm{E}$ and $\mathrm{C}$, beta-carotene, and other carotenoids. Ann N Y Acad Sci. 1992;669:7-20.

22. Shacter E, Beecham EJ, Covey JM, Kohn KW, Potter M. Activated neutrophils induce prolonged DNA damage in neighboring cells. Carcinogenesis. 1988;9(12):2297-304.

23. Kong Y, Trabucco SE, Zhang H. Oxidative stress, mitochondrial dysfunction and the mitochondria theory of aging. Interdiscip Top Gerontol. 2014;39:86-107.

24. Linnane AW, Marzuki S, Ozawa T, Tanaka M. Mitochondrial DNA mutations as an important contributor to ageing and degenerative diseases. Lancet. 1989;1 (8639):642-5.

25. Pérez VI, Van Remmen H, Bokov A, Epstein CJ, Vijg J, Richardson A. The overexpression of major antioxidant enzymes does not extend the lifespan of mice. Aging Cell.
2009;8(1):73-5.

26. Chiou JF, Hu ML. Elevated lipid peroxidation and disturbed antioxidant enzyme activities in plasma and erythrocytes of patients with uterine cervicitis and myoma. Clin Biochem. 1999;32(3):189-92.

27. Santulli P, Borghese B, Lemaréchal H, Leconte M, Millischer AE, Batteux F et al. Increased serum oxidative stress markers in women with uterine leiomyoma. PLoS One 2013;8(8):e72069.

28. Vural M, Camuzcuoglu H, Toy H, Camuzcuoglu A, Aksoy $\mathrm{N}$. Oxidative stress and prolidase activity in women with uterine fibroids. J Obstet Gynaecol. 2012;32(1):68-72.

29. Yucel A, Sanhal CY, Daglar K, Kara O, Uygur D, Erel O. Thiol/disulphide homeostasis in pregnant women with Familial Mediterranean fever. Redox Rep. 2016;21(6): 287-91.

30. Vignini A, Sabbatinelli J, Clemente N, Delli Carpini G, Tassetti M, Zagaglia G, et al. Preperitoneal fat thicknesses, lipid profile, and oxidative status in women with uterine fibroids. Reprod Sci. 2017;24(10):1419-25.

31. Markowska A, Mardas M, Gajdzik E, Zagrodzki P, Markowska J. Oxidative stres markers in uterine fibroids tissue in pre- and postmenopausal women. Clin Exp Obstet Gynecol. 2015;42(6):725-9.

32. Caglayan A, Katlan DC, Tuncer ZS, Yuce K, Sayal HB, Kocer-Gumusel B. Assessment of oxidant-antioxidant status alterations with tumor biomarkers and reproductive system hormones in uterine myomas. Eur J Obstet Gynecol Reprod Biol. 2018;229:1-7.

33. Fletcher NM, Saed MG, Abu-Soud HM, Al-Hendy A, Diamond MP, Saed GM. Uterine fibroids are characterized by an impaired antioxidant cellular system: potential role of hypoxia in the pathophysiology of uterine fibroids. J Assist Reprod Genet. 2013;30(7):969-74.

34. Wise LA, Laughlin-Tommaso SK. Epidemiology of uterine fibroids: from menarche to menopause. Clin Obstet Gynecol. 2016;59(1):2-24.

35. Ishikawa H, Ishi K, Serna VA, Kakazu R, Bulun SE, Kurita T. Progesterone is essential for maintenance and growth of uterine leiomyoma. Endocrinology. 2010;151 (6):2433-42.

36. Farías JG, Molina VM, Carrasco RA, Zepeda AB, Figueroa E, Letelier P et al. Antioxidant therapeutic strategies for cardiovascular conditions associated with oxidative stress. Nutrients. 2017;9(9).

37. Mut-Salud N, Álvarez PJ, Garrido JM, Carrasco E, Aránega A, Rodríguez-Serrano F. Antioxidant intake and antitumor therapy: toward nutritional recommendations for optimal results. Oxid Med Cell Longev. 2016;2016: 6719534.

38. Kontic-Vucinic O, Terzic M, Radunovic N. The role of antioxidant vitamins in hypertensive disorders of pregnancy. J Perinat Med. 2008;36(4):282-90. 